\title{
Analysis of JPSS J1 VIIRS Polarization Sensitivity Using the NIST T-SIRCUS
}

\author{
Jeff McIntire $^{a}$, James B. Young ${ }^{b}$, David Moyer ${ }^{c}$, Eugene Waluschka ${ }^{d}$, Hassan Oudrari ${ }^{a}$, and \\ Xiaoxiong Xiong ${ }^{d}$ \\ ${ }^{a}$ Science Systems and Applications, Inc., Lanham, MD 20706 USA \\ ${ }^{b}$ Stellar Solutions, Palo Alto, CA 94306 USA \\ ${ }^{c}$ The Aerospace Corporation, El Segundo, CA 90245-4691 USA \\ ${ }^{d}$ NASA Goddard Space Flight Center, Greenbelt, MD, 20771 USA
}

\begin{abstract}
The polarization sensitivity of the Joint Polar Satellite System (JPSS) J1 Visible Infrared Imaging Radiometer Suite (VIIRS) measured pre-launch using a broadband source was observed to be larger than expected for many reflective bands. Ray trace modeling predicted that the observed polarization sensitivity was the result of larger diattenuation at the edges of the focal plane filter spectral bandpass. Additional ground measurements were performed using a monochromatic source (the NIST T-SIRCUS) to input linearly polarized light at a number of wavelengths across the bandpass of two VIIRS spectral bands and two scan angles. This work describes the data processing, analysis, and results derived from the T-SIRCUS measurements, comparing them with broadband measurements. Results have shown that the observed degree of linear polarization, when weighted by the sensor's spectral response function, is generally larger on the edges and smaller in the center of the spectral bandpass, as predicted. However, phase angle changes in the center of the bandpass differ between model and measurement. Integration of the monochromatic polarization sensitivity over wavelength produced results consistent with the broadband source measurements, for all cases considered.
\end{abstract}

Keywords: JPSS, S-NPP, VIIRS, polarization, calibration

\section{INTRODUCTION}

The Joint Polar Satellite System (JPSS) J1 Visible Infrared Imaging Radiometer Suite (VIIRS) instrument is the second VIIRS sensor [the first being the Suomi National Polar-orbiting Partnership (S-NPP) VIIRS ${ }^{1,2}$ ] and is currently undergoing pre-launch testing with launch scheduled for late $2016 .{ }^{3}$ The polarization sensitivity of J1 VIIRS, measured pre-launch for the visible and near-infrared spectral bands (which cover the spectral region from $\sim 400-900 \mathrm{~nm}$ ), was found to be larger than observed for a number of S-NPP VIIRS bands. The bluest spectral bands showed the largest polarization sensitivities for J1 VIIRS, with up to $6.4 \%$ for the $412 \mathrm{~nm}$ band; ${ }^{4}$ in comparison, the same band on S-NPP was observed to have a polarization sensitivity of less than $\sim 3 \%{ }^{5}$ In addition, large detector-to-detector, half angle mirror (HAM) side, and scan angle dependence were observed in J1 testing. While similar characteristics were observed for S-NPP, it was to a much lesser extent. ${ }^{5}$

The pre-launch testing used to characterize the J1 polarization sensitivity was conducted with a broadband source in a setup that was nearly identical to that used for S-NPP. ${ }^{4-6}$ However, these tests provided limited information on the root cause of this sensitivity. Modeling based on limited component level measurements of the optics (developed by Raytheon, using the FRED ray tracing software ${ }^{6}$ ) predicted that the polarization sensitivity was caused by larger diattenuation on the edges of the spectral bandpass. Additional polarization sensitivity measurements using a monochromatic source [the NIST T-SIRCUS (Traveling Spectral Irradiance and Radiance Responsivity Calibrations using Uniform Sources) ${ }^{7}$ ] were performed to validate this hypothesis. This work will detail the analysis and results of the T-SIRCUS measurements, and compare to the broadband measurements and model predictions.

Send correspondence to Jeff McIntire: jeffrey.mcintire@ssaihq.com 


\section{TEST OVERVIEW}

As the monochromatic polarization testing was exploratory, and not included in the baseline pre-launch performance testing, a limited number of measurements were performed. ${ }^{6}$ The spectral bands selected for testing were M1 (band center $\sim 412 \mathrm{~nm}$ ) and M4 (band center $\sim 555 \mathrm{~nm}$ ). Band M1 showed the largest polarization sensitivity of any J1 VIIRS bands, up to $\sim 6.4 \%$, in addition to large detector-to-detector, HAM side, and scan angle dependence. Model predictions were generally consistent with the observed measurements for M1. Band M4 was observed to have polarization sensitivity of up to $\sim 4.3 \%$ as well as significant detector-to-detector and HAM side dependence. However, for M4, the model predictions did not reproduce the observed behavior well.

\subsection{T-SIRCUS}

NIST (National Institute of Standards and Technology) has developed a laser-based facility known as SIRCUS for use in radiance and irradiance responsivity calibration. ${ }^{7}$ A portable version known as T-SIRCUS (or Traveling SIRCUS) was transported to the Raytheon facility in El Segundo, CA during December 2014 for J1 VIIRS instrument level testing. SIRCUS consists of a series of tunable lasers; for polarization testing, the frequencydoubled output from a custom optical parametric oscillator pumped at $532 \mathrm{~nm}$ by a $\mathrm{Nd}: \mathrm{YVO}_{4}$ laser was used to produce monochromatic illumination from $397 \mathrm{~nm}-424 \mathrm{~nm}$ and from $543 \mathrm{~nm}-565 \mathrm{~nm}$ while a Rhodamine $6 \mathrm{G}$ dye laser was used to produce monochromatic illumination from $566 \mathrm{~nm}$ to $572 \mathrm{~nm}$. The bandwidth of the optical parametric oscillator was $\sim 0.02 \mathrm{~nm}$ in the $400 \mathrm{~nm}$ region and $\sim 0.03 \mathrm{~nm}$ in the $550 \mathrm{~nm}$ region; the bandwidth of the dye laser was also $\sim 0.02 \mathrm{~nm} .^{6}$

\subsection{Test Setup}

The T-SIRCUS lasers, via fiber optics, fed a $1 \mathrm{~m}$ NIST integrating sphere with a 12 in circular aperture (a radiance monitor was used to track the radiance output from the sphere in real-time) ${ }^{6}$ Light exiting this integrating sphere illuminated a sheet polarizer (BVONIR) mounted in a rotating stage, which could be rotated from 0 to 360 degrees. The now polarized light next entered VIIRS telescope aperture. Additional elements were added to the path for supplemental stray light and polarizer efficiency tests. Two stray light tests were conducted: with a "lollipop" obscuration inserted into the path between the integrating sphere and the rotating sheet polarizer, and with the source off. The efficiency of the sheet polarizer was measured by inserting an additional fixed BVONIR polarizer into the path between the rotating polarizer and VIIRS aperture. Various baffling was used to minimize contamination for other sources or paths.

The VIIRS instrument was set to operational mode with the telescope fixed, staring at the source. Bands M1 and M4 were operated in fixed high gain while the Day Night Band (DNB) was in auto gain (DNB data is not discussed in this work). VIIRS was mounted on a rotary table, such that the instrument could view the source from different scan angles; for T-SIRCUS testing, VIIRS was positioned to view the source at -8 and +45 degrees scan angles.

For all tests (stray light, efficiency, and sensitivity), the polarizer sheet was rotated from 0 to 180 degrees in 15 degree increments. The polarizer sheet dwelled at every angle for a given amount of time (45 or 75 seconds, depending on the test) before transitioning to the next polarizer angle. A shutter on the source was used to provide a dark offset correction; the shutter cycle was 18 or 30 seconds, depending on the test.

\section{METHODOLOGY}

\subsection{Fourier Analysis}

The polarization sensitivity measurements made using T-SIRCUS were analyzed using Fourier analysis. ${ }^{8}$ The offset corrected sensor response $(d n)$ at a given wavelength $(\lambda)$ and polarization angle $(\alpha)$ was modeled as a fourth order Fourier expansion expressed by

$$
d n(\alpha, \lambda)=\frac{1}{2} c_{0}(\lambda)+\sum_{n=1}^{4}\left[c_{n}(\lambda) \cos (n \alpha)+d_{n}(\lambda) \sin (n \alpha)\right]
$$


where the zeroth and second order Fourier coefficients are given by

$$
\begin{gathered}
\frac{1}{2} c_{0}(\lambda)=\frac{1}{\pi} \int_{0}^{\pi} d n(\theta, \lambda) d \theta \\
C_{2}(\lambda)=\frac{2 c_{2}(\lambda)}{c_{0}(\lambda)}=\frac{4}{\pi c_{0}(\lambda)} \int_{0}^{\pi} d n(\theta, \lambda) \cos (2 \theta) d \theta \\
D_{2}(\lambda)=\frac{2 d_{2}(\lambda)}{c_{0}(\lambda)}=\frac{4}{\pi c_{0}(\lambda)} \int_{0}^{\pi} d n(\theta, \lambda) \sin (2 \theta) d \theta
\end{gathered}
$$

Note that the limits on the integrals reflect the extent of the measured polarizer angles, 0 to $\pi$. The first, third, and fourth order Fourier coefficients are not considered in this work, other than to state that they are in general subdominant. The Fourier expansion given in Eq. (1) can be rewritten as

$$
d n(\alpha, \lambda)=\frac{1}{2} c_{0}(\lambda)\left\{1+\sum_{n=1}^{4} a_{n}(\lambda) \cos \left[n \alpha+\delta_{n}(\lambda)\right]\right\}
$$

where

$$
\begin{aligned}
& a_{n}(\lambda)=\frac{\sqrt{C_{n}^{2}(\lambda)+D_{n}^{2}(\lambda)}}{\sqrt{a_{2}^{e f f}(\lambda)}} \\
& \delta_{n}(\lambda)=\frac{1}{2} \arctan \left[\frac{D_{n}(\lambda)}{C_{n}(\lambda)}\right]
\end{aligned}
$$

Here $a_{2}^{\text {eff }}$ is the polarizer efficiency determined from Eq. (6) without the denominator using the cross polarizer data. Here $a_{2}$ is the degree of linear polarization (DoLP) and $\delta_{2}$ is the phase angle.

\subsection{Band Dependent Polarization Sensitivity}

In order to compare to the broadband measurements of the polarization sensitivity, ${ }^{4,6}$ the Fourier analysis described above is averaged over the spectral bandpass, weighted by the broadband input spectrum and spectral transmittance of the sensor, or

$$
\begin{aligned}
& C_{2}(B)=\int C_{2}(\lambda) R S R(\lambda) d \lambda \\
& D_{2}(B)=\int D_{2}(\lambda) R S R(\lambda) d \lambda
\end{aligned}
$$

where $B$ indicates a band-dependent quantity. Here $R S R$ denotes the spectral transmittance weighted by the broadband source profile. For band M1, this also includes the transmittance of a long-wave blocking filter, included to reduce the effects of out-of-band leaks in the broadband measurements. ${ }^{4,6}$ The Fourier coefficients generated from Eqs. (3) and (4) were resampled to $1 \mathrm{~nm}$ from the measured T-SIRCUS wavelengths. Next the band dependent polarization factor (DoLP) and phase are defined as

$$
\begin{aligned}
& a_{2}(B)=\frac{\sqrt{C_{2}^{2}(B)+D_{2}^{2}(B)}}{\sqrt{a_{2}^{\text {eff }}(B)}} \\
& \delta_{2}(B)=\frac{1}{2} \arctan \left[\frac{D_{2}(B)}{C_{2}(B)}\right]
\end{aligned}
$$

For clarity in the plotting, here $0 \leq \delta_{2}(B)<\pi$. The above analysis was also performed on the ray trace model data. 


\section{ANALYSIS RESULTS}

\subsection{Data Quality and Reduction}

The test data acquired during T-SIRCUS polarization testing is listed in Table 1 including the wavelength, target band, HAM side, scan angle, and test type. For each wavelength, the polarizer was rotated from 0 to 180 degrees in 15 degree increments. For every polarizer angle measured, the polarizer dwelled for either 45 or 75 seconds (depending on the test). During that time, the shutter on the T-SIRCUS was either open or closed. The time stamps for both the shutter state and polarizer dwell were recorded; these time stamps were matched to VIIRS scan times to determine which scans corresponded to shutter open and which to shutter closed for a given polarizer angle. The data was also screened for wavelength; if the laser wavelength drifted by more than $0.15 \mathrm{~nm}$, the corresponding scans were discarded. Additionally, the data was further screened to eliminate scans during which the shutter state changed within a scan (observed through higher standard deviations over pixels or if the average $d n$ differed significantly from other scans with the same shutter state). Note that for some polarizer angle - wavelength combinations, no valid scans remained after the screening.

VIIRS was in operational day mode during the T-SIRCUS polarization testing. Thus for every scan, 6304 pixels were recorded for each detector. The average response and standard deviation were determined for each scan and detector, using an outlier rejection. Once the shutter open and closed scan were selected for a given polarizer angle and wavelength, the $d n$ was determined by subtracting the average response of the shutter closed scans from the average response of the shutter open scans.

Table 1. T-SIRCUS polarization measurements performed as a function of wavelength, HAM side, scan angle, and test type (stray light, polarizer efficiency, or polarization sensitivity). Wavelengths were rounded to the nearest nm.

\begin{tabular}{|c|c|c|c|c|}
\hline Test Type & $\begin{array}{l}\text { HAM } \\
\text { Side }\end{array}$ & $\begin{array}{l}\text { Scan Angle } \\
\text { [degrees] }\end{array}$ & $\begin{array}{l}\text { Target } \\
\text { Band }\end{array}$ & $\begin{array}{l}\text { Wavelengths } \\
{[\mathrm{nm}]}\end{array}$ \\
\hline Stray Light - Dark & 1 & -8 & M1, M4 & NA \\
\hline Stray Light - Lollipop & 1 & -8 & $\begin{array}{l}\text { M1 } \\
\text { M4 }\end{array}$ & $\begin{array}{l}415 \\
559\end{array}$ \\
\hline $\begin{array}{l}\text { Polarizer } \\
\text { Efficiency }\end{array}$ & 1 & -8 & $\begin{array}{l}\text { M1 } \\
\text { M4 }\end{array}$ & $\begin{array}{l}401,412,420 \\
559\end{array}$ \\
\hline $\begin{array}{c}\text { Polarization } \\
\text { Sensitivity }\end{array}$ & 1 & -8 & M1 & $\begin{array}{l}397,400,402,404,406,408,410,413,415, \\
417,419,421,424\end{array}$ \\
\hline $\begin{array}{l}\text { Polarization } \\
\text { Sensitivity }\end{array}$ & 1 & +45 & M1 & $\begin{array}{l}397,399,402,404,406,408,410,413,415, \\
417,419,421,424\end{array}$ \\
\hline $\begin{array}{c}\text { Polarization } \\
\text { Sensitivity }\end{array}$ & 1 & -8 & M4 & $\begin{array}{l}543,546,547,548,550,552,553,555,556, \\
558,560,561,562,564,567,569,572\end{array}$ \\
\hline $\begin{array}{l}\text { Polarization } \\
\text { Sensitivity }\end{array}$ & 0 & -8 & M4 & $\begin{array}{l}543,545,547,548,550,553,556,559,562, \\
564,567,569,572\end{array}$ \\
\hline $\begin{array}{c}\text { Polarization } \\
\text { Sensitivity }\end{array}$ & 1 & +45 & M4 & $\begin{array}{l}543,545,547,549,551,552,553,554,556, \\
558,559,561,562,564,567,569,572\end{array}$ \\
\hline
\end{tabular}

The thirteen measurements per wavelength (assuming there is valid data for all polarizer angles) were used to determine the Fourier coefficients in Eqs. $(2)-(4)$. For the wavelengths where one or more polarizer angles did not have any valid data, some approximations were made to replace the missing data: data from 0 degrees was used to replace missing data at 180 degrees and vice versa; if one polarizer angle was missing, then a linear interpolation across the interval was made. For the remaining cases, a function fit was used to determine the 
(a)

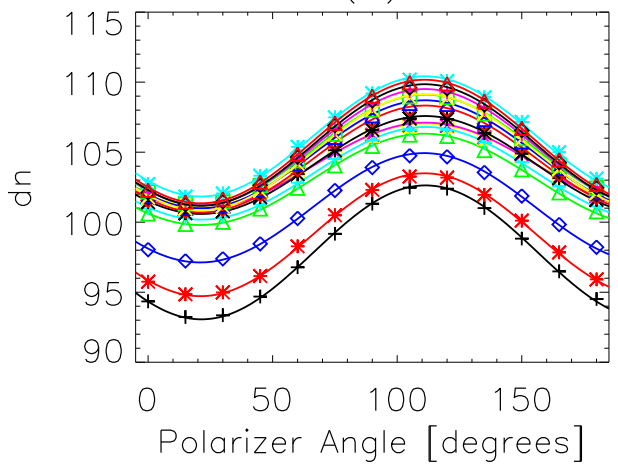

(c)

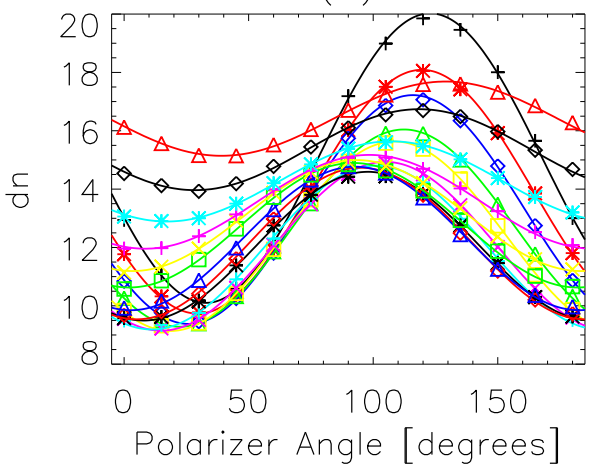

(b)

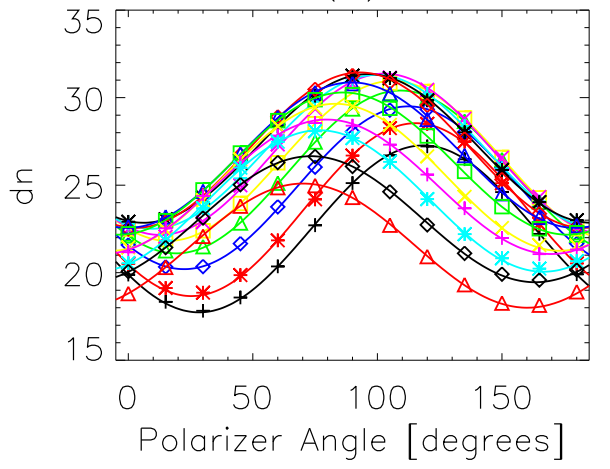

(d)

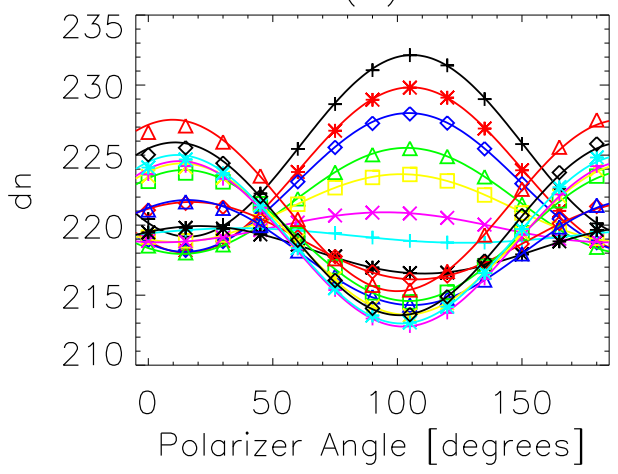

Figure 1. Measured $d n$ versus polarizer angle [degrees] for M1 at $406 \mathrm{~nm}$ (a), M1 at $421 \mathrm{~nm}$ (b), M4 at $543 \mathrm{~nm}$ (c), and M4 at $556 \mathrm{~nm}$ (d) at -8 degrees scan angle, HAM side 1. The legend defines the different symbol / color combinations which correspond to each detector.

zeroth and second order Fourier coefficients. The wavelength dependent Fourier coefficients defined in Eqs. (3) and (4) were resampled to $1 \mathrm{~nm}$, then the DoLP and phase were determined from Eqs. (6) and (7).

For the comparisons to the broadband measurements, the resampled Fourier coefficients were integrated over the spectral bandpass, as defined by Eqs. (8) and (9). For M4, the spectral transmittance of VIIRS was convolved with the broadband source profile and renormalized; for M1, the spectral transmittance of VIIRS was convolved with the broadband source profile as well as the transmittance of a long-wave blocking filter and renormalized. Finally, the band dependent DoLP and phase were determined from Eqs. (10) and (11). The average polarizer efficiency for a given band was used in Eq. (10).

\subsection{Results}

The measured $d n$ for each polarizer angle at $406 \mathrm{~nm}$ is shown in Figure 1(a) for all detectors (at -8 degrees scan angle, HAM side 1); the symbols represent the measured $d n$ and the solid lines denote the functions defined in Eq. (5). Each detector is represented by a different symbol / color as defined in the legend. Similarly, the measurements and fits for $421 \mathrm{~nm}, 543 \mathrm{~nm}$, and $556 \mathrm{~nm}$ (at -8 degrees scan angle, HAM side 1) are shown by the symbols and solid lines in Figures $1(\mathrm{~b})-1(\mathrm{~d})$. Note that the lines fit the data very well and closely follow a two-cycle oscillation. The second order Fourier components dominate in all cases except where the second order components become very small. In addition, the DoLP and phase may vary considerably between detectors.

The DoLP for M1 (-8 degrees scan angle, HAM side 1, resampled to $1 \mathrm{~nm}$ ) is shown in Figure 2(a); here the DoLP becomes larger as one moves away from the center of the bandpass and the DoLP is as large as $80 \%$. However, the wavelength regions with large polarization sensitivity in Figure 2(a) correspond to the edges of the 


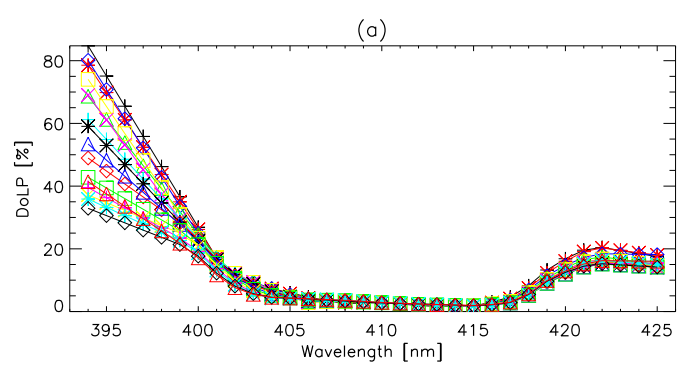

(c)

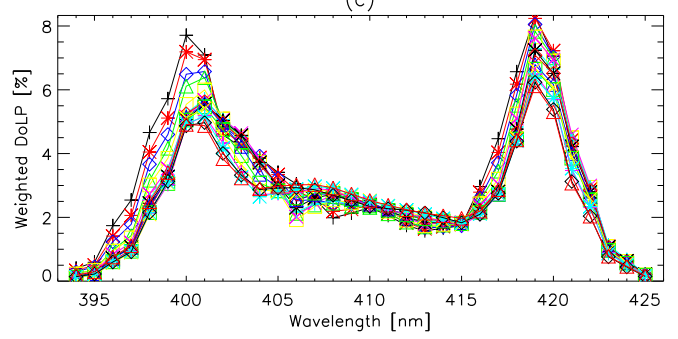

(e)

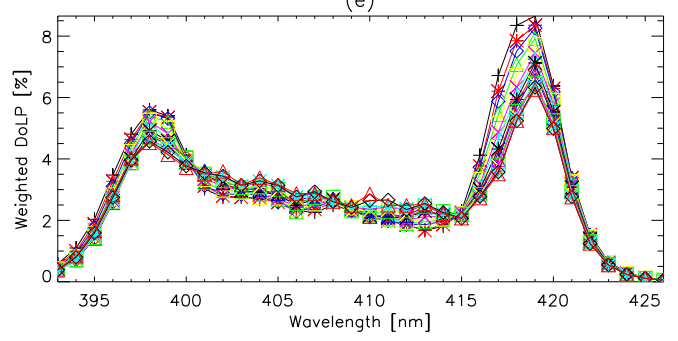

(b)

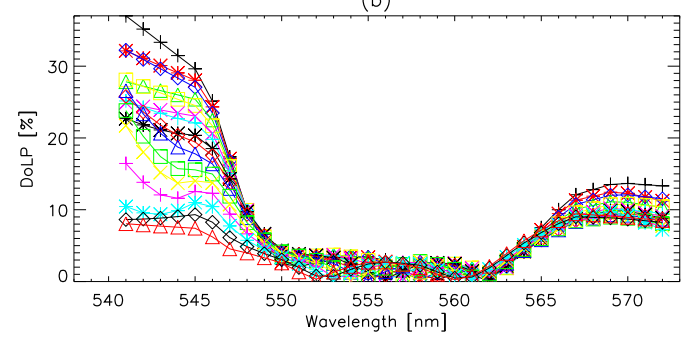

(d)

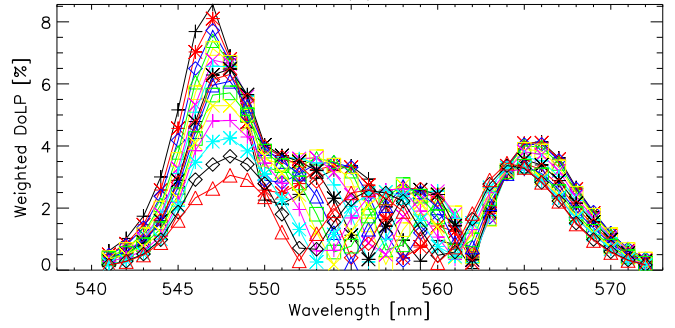

(f)

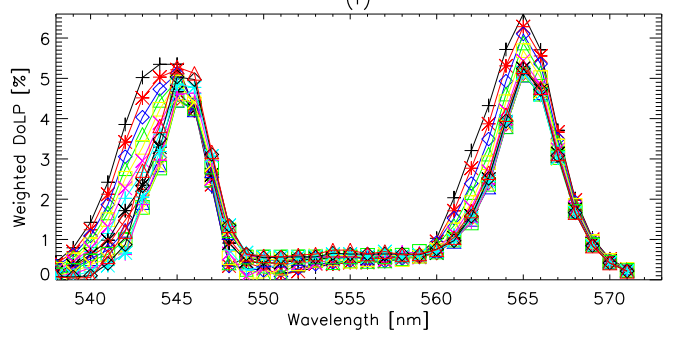

Figure 2. Measured DoLP versus wavelength for M1 (a) and M4 (b); measured weighted DoLP versus wavelength for M1 (c) and M4 (d); modeled weighted DoLP versus wavelength for M1 (e) and M4 (f). All plots show data at -8 degrees scan angle, HAM side 1. The legend defines the different symbol / color combinations which correspond to each detector.

spectral bandpass; weighting the DoLP by the spectral transmittance of the system, as shown in Figure 2(c), indicates that the DoLP increases when one gets to the sides of the bandpass, and then decreases in the middle. Similar plots for M4 (-8 degrees scan angle, HAM side 1, resampled to $1 \mathrm{~nm}$ ) are shown in Figures 2(b) and 2(d). For the M4 case, the DoLP oscillates in the center of the bandpass; the minimums in DoLP correspond to phase angle shifts in the polarization sensitivity. The cases not shown (M1, +45 degrees scan angle, HAM side 1; M4, -8 degrees scan angle, HAM side 0; and M4, +45 degrees scan angle, HAM side 1) show similar results. The corresponding ray trace model results are shown in Figures 2(e) and 2(f) for M1 and M4 (-8 degrees scan angle, HAM side 1). Note that the model and measurements agree in the general shape and characteristics of the DoLP versus wavelength. For M1, the modeled bandpass is slightly wider than the measurement; for M4, the modeled bandpass is also wider and the phase angle shifts that occur in the center of the bandpass were not captured in the model.

The band dependent DoLP and phase angles are shown for all measured cases in Figure 3. In general, the T-SIRCUS results agree reasonably well with the broadband measurements. There are differences in the DoLP for both the M1 and M4 cases of up to $0.5 \%$ compared to the broadband measurements. Note that the M1 and M4 bandpasses were not critically sampled (the laser bandpass was less than 1 nm). Model differences with measurements are small for M1, but larger for M4; for M4, the detector dependence was not captured well by the model. This may result from the model not predicting the phase angle shifts occurring in the center of the M4 bandpass. The phase angles are very similar for the M1 cases; however, for M4, the phase angle differences between T-SIRCUS and broadband measurements were larger for higher number detectors, up to $\sim 14$ degrees. 
(a)

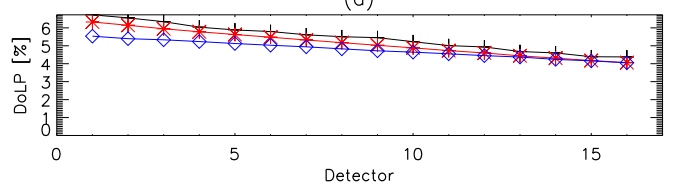

(c)

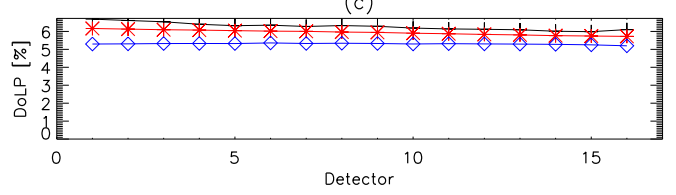

(e)

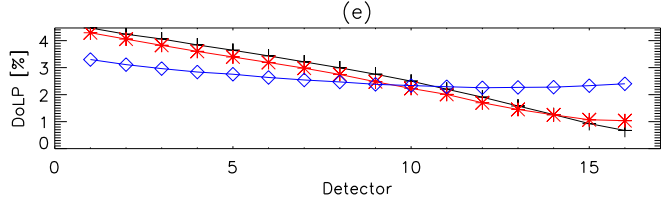

(g)

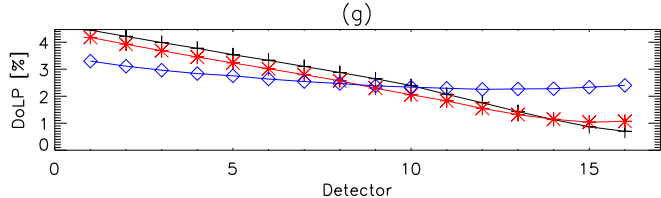

(i)

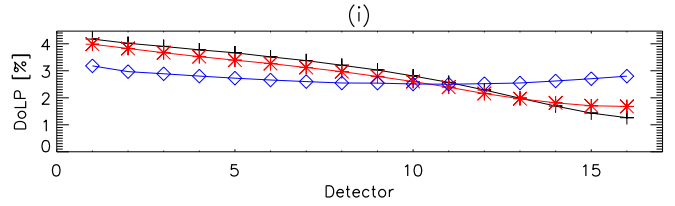

(b)

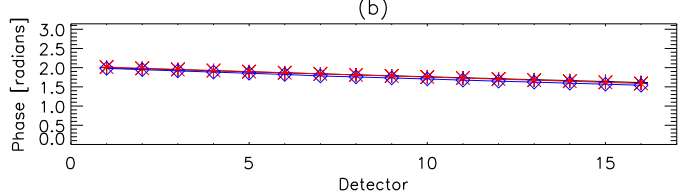

(d)

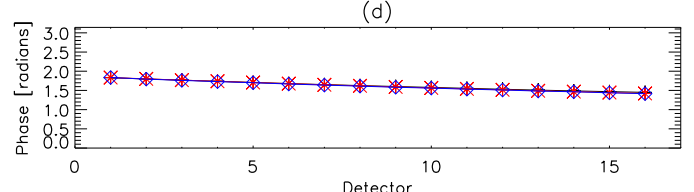

(f)

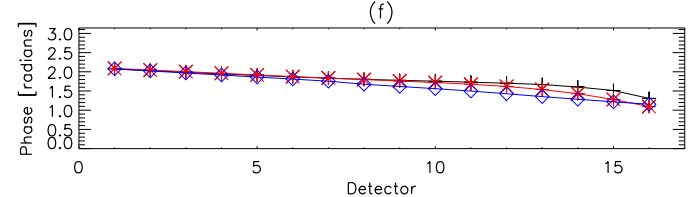

(h)

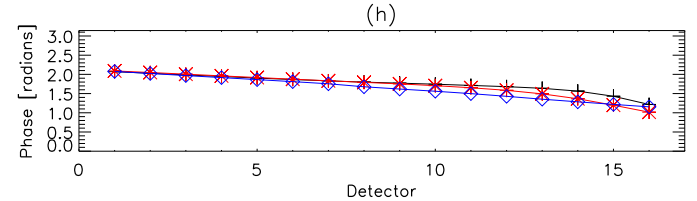

(j)

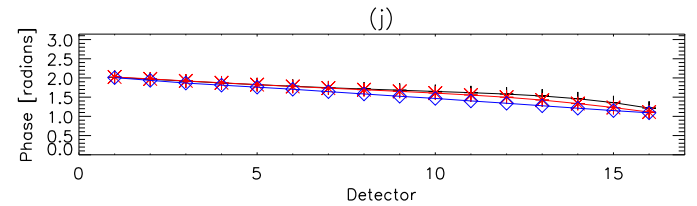

Figure 3. Plots of the DoLP and phase showing: M1 at -8 degrees scan angle, HAM side 1 in (a) and (b); M1 at +45 degrees scan angle, HAM side 1 in (c) and (d); M4 at -8 degrees scan angle, HAM side 1 in (e) and (f); M4 at -8 degrees scan angle, HAM side 0 in $(\mathrm{g})$ and $(\mathrm{h}) ; \mathrm{M} 4$ at +45 degrees scan angle, HAM side 1 in (i) and (j). Black '+' indicates T-SIRCUS measurements, red ' $*$ ' denote broadband measurements, and blue ' $\diamond$ ' refer to the model.

\section{CONCLUSIONS}

Broadband measurements of the JPSS J1 VIIRS instrument showed larger than expected polarization sensitivity in the bluer bands (up to $\sim 6.4 \%$, compared to less than $\sim 3 \%$ for S-NPP VIIRS). A Raytheon developed ray trace model, based on limited component level measurements of the optics, indicated that the larger sensitivity was the result of large diattenuation on the edges the bandpass. In part to investigate the model predictions, monochromatic measurements of the polarization sensitivity of select JPSS J1 VIIRS bands (M1 and M4) were performed at the Raytheon El Segundo, CA facility in December 2014 using the NIST T-SIRCUS. The monochromatic measurements largely validated the model predictions: larger sensitivity was observed on the edges of the bandpass relative to the center of the bandpass. In addition, when averaged over the bandpass, the T-SIRCUS measurements were consistent with the broadband measurements to within $\sim 0.5 \%$ (even with the bandpasses under sampled). There were some differences with the model; in particular, the model did not capture the phase angle shifts in the center of the M4 bandpass. These monochromatic measurements successfully demonstrated the possible future testing on forthcoming instruments (JPSS J2+) as well as the need for component level measurements useful in the ray trace modeling.

\section{ACKNOWLEDGMENTS}

The authors would like to acknowledge the following: Steve Brown and Keith Lykke from NIST and Brendan McAndrew from NASA for setting up and operating the T-SIRCUS lasers during testing, Joel McCorkel from 
NASA for organizing the testing, and the Raytheon test team including Tung Wang for conducting the test. The above mentioned proved valuable information and support to the analysis presented in this work.

\section{REFERENCES}

[1] Oudrari, H., McIntire, J., Xiong, X., Butler, J., Lee, S., Lei, N., Schwarting, T., and Sun, J., "Prelaunch radiometric characterization and calibration of the S-NPP VIIRS sensor," IEEE T. Geosci. Remote 53(4), $2195(2015)$.

[2] Xiong, X., Butler, J., Chiang, K., Efremova, B., Fulbright, J., Lei, N., McIntire, J., Oudrari, H., Sun, J., Wang, Z., and Wu, A., "VIIRS on-orbit calibration methodology and performance," J. Geophys. ResAtmos. 119(9), 5065 (2014).

[3] Oudrari, H., McIntire, J., Xiong, X., Butler, J., Efremova, B., Ji, Q., Lee, S., and Schwarting, T., "Preliminary assessment of JPSS-1 VIIRS pre-launch radiometric performance," Proc. of SPIE 9607 (2015). In press.

[4] Moyer, D., McIntire, J., Waluschka, E., Xiong, X., and DeLuccia, F., "JPSS-1 VIIRS pre-launch polarization testing and performance," IEEE T. Geosci. Remote (2015). In press.

[5] Sun, J., "Polarization sensitivity analysis of VIIRS F1 VisNIR bands," Unpublished (2010).

[6] Waluschka, E., McCorkel, J., McIntire, J., Moyer, D., McAndrew, B., Brown, S. W., Lykke, K., Young, J. B., Fest, E., Butler, J., Wang, T. R., Monroy, E. O., Turpie, K., Meister, G., and Thome, K. J., "VIIRS polarization narrative," Proc. of SPIE 9607 (2015). In press.

[7] Brown, S. W., Eppeldauer, G. P., and Lykke, K. R., "Facility for spectral irradiance and radiance responsivity calibrations using uniform sources," Appl. Opt. 45(32), 8218-8237 (2006).

[8] Boas, M. L., [Mathematical Methods in the Physical Sciences], John Wiley and Sons (1985). 